\section{Self control}

Ten per cent of our adult bone mass is replaced each year - a constant remodelling process in which osteoclasts, cells that destroy and resorb old bone, and osteoblasts, which deposit new bone in its place, have a key role. Several molecules have been proposed to coordinate the activities of osteoblasts and osteoclasts, but as a recent report in Nature reveals, osteoclasts might also keep themselves under strict control. Targeted use of the key molecule responsible - interferon- $\beta$ (IFN- $\beta$ ) - might be of benefit in diseases characterized by inappropriately high bone resorption, such as osteoporosis and rheumatoid arthritis.

Osteoclast maturation from precursors to functional bone-resorbing cells is stimulated when a protein on the surface of precursors — RANK - is activated by its ligand RANKL, which is usually present on the surface of osteoblasts. Activation of RANK leads to an increase in the expression of certain genes, including that coding for the transcription factor c-Fos, which is known to be crucial for osteoclast differentiation.
How are the effects of RANKL regulated? Various molecules are known to control the level and availability of RANKL on osteoblast surfaces; however, it was not known whether RANKL signalling itself activates a negative regulatory mechanism to maintain bone homeostasis. To answer this question, Takayanagi et al. set out to identify genes induced when RANKL binds to RANK. Initial observations highlighted the IFN- $\beta$ signalling pathway, and its key role was confirmed when the authors showed that mice lacking IFN- $\beta$ or its cell-surface receptor have low bone mass and increased bone resorption by osteoclasts.

Further experiments indicated that the IFN- $\beta$ gene is induced by $c$-Fos, and that IFN- $\beta$ inhibits RANKL-induced osteoclast differentiation by inhibiting the expression of c-Fos. Bringing all the observations together, it seems that IFN- $\beta$ and c-Fos are part of a negative feedback loop initiated by RANKL. In one half of the loop, RANK induces IFN- $\beta$ expression through c-Fos. In the other half, secreted IFN- $\beta$ binds to its receptor on osteoclast precursors, which

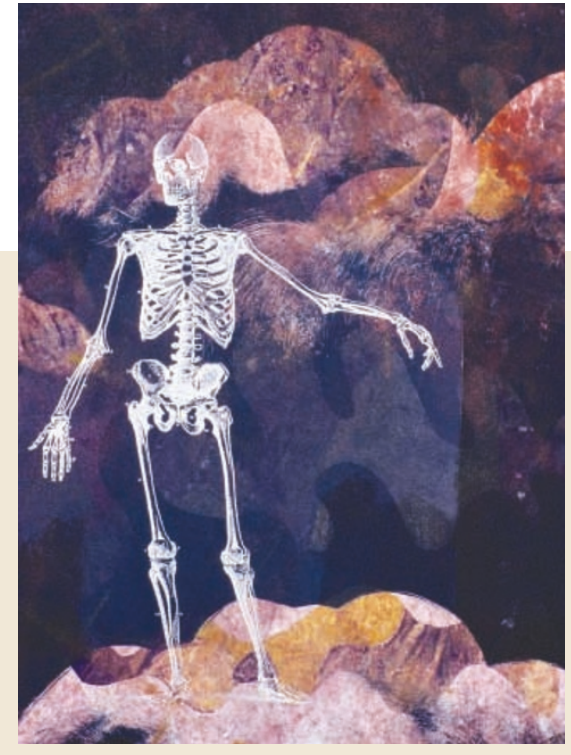

causes a decrease in c-Fos levels, and thus inhibition of osteoclast differentiation. So, could manipulating this loop be of therapeutic benefit? Initial indications seem encouraging - the authors showed that IFN- $\beta$ prevented bone resorption in a mouse model of arthritis, and intriguingly, some patients with multiple sclerosis being treated with IFN- $\beta$ have been reported to show improvements in their rheumatoid arthritis.

Peter Kirkpatrick

(2) References and links ORIGINAL RESEARCH PAPER Takayanagi, H. et al. RANKL maintains bone homeostasis through c-Fosdependent induction of interferon- $\beta$. Nature 416, 744-749 (2002)

Encyclopedia of Life Sciences: http://www.els.net regeneration of bone

\title{
The nature of the beast
}

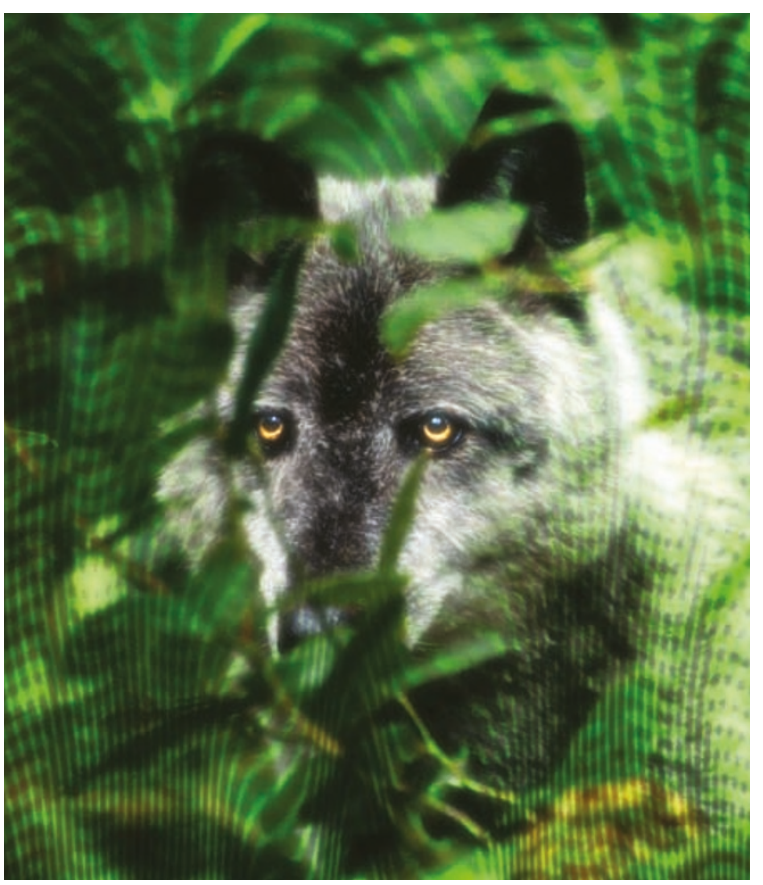

The deposition of insoluble amyloid fibrils is a well-characterized part of the pathogenesis of more than 100 diseases, but relatively little is known about the mechanisms that underlie fibril formation. Back-to-back articles in the May issue of Nature Structural Biology now give us snapshots of two stages in the pathway to these familiar enemies.

Both studies used NMR spectroscopy to investigate the misfolding and aggregation of $\beta_{2}$-microglobulin $(\beta 2 \mathrm{M})$. Although it is normally soluble, $\beta 2 \mathrm{M}$ is found at a high concentration in the amyloid plaques that can form in the musculoskeletal system of patients with chronic renal failure who are receiving long-term haemodialysis. The $\beta 2 \mathrm{M}$ protein has previously been shown to form amyloid-like fibrils spontaneously under denaturing conditions, and it was an early precursor of these fibrils that was imaged by Radford and colleagues. The study found that $\beta 2 \mathrm{M}$ amyloid precursors partially retain the double $\beta$-sheet structure that is characteristic of the native protein, which implies that these $\beta$-sheets might contribute to the cross- $\beta$-sheets that are characteristic of mature amyloid fibrils.

By contrast, Gotto and colleagues studied mature $\beta 2 \mathrm{M}$ fibrils, and developed an extension of the hydrogen/deuterium-exchange method to explore the structure of the interior of fibrils at single-residue resolution. At the core of the fibril, they describe an extensively hydrogenbonded $\beta$-sheet, accounting for the great stability of amyloid fibrils.

Recent evidence that implicates early intermediates in the pathway to fibril formation as being culprits in the damage that is caused by amyloid diseases (see, for instance, the further reading below) serves to underscore the importance of understanding not just the nature of the fibrils, but also the stages that lead to their creation.

Adam Smith

\section{(2) References and links}

ORIGINAL RESEARCH PAPER McParland, V. J. et al.

Structural properties of an amyloid precursor of $\beta_{2}$

microglobulin. Nature Struct. Biol. 9, 326-331 (2002) | Hoshino, M. et al. Mapping the core of the $\beta$-microglobulin amyloid fibril by H/D exchange. Nature Struct. Biol. 9, 332-336 (2002) FURTHER READING Bucciantini, M. et al. Inherent toxicity of aggregates implies a common mechanism for protein misfolding diseases. Nature 416, 507-511 (2002) |Walsh, D. M. et al. Naturally secreted oligomers of amyloid $\beta$ protein potently inhibit hippocampal long-term potentiation in vivo. Nature 416 535-539 (2002) 